\title{
Induction Chemotherapy of Docetaxel and Cisplatin for the Elderly Patients with Squamous Cell Carcinoma of the Head and Neck
}

Young-Jin Choi, M.D. ${ }^{1}$, Jooseop Chung, M.D. ${ }^{1}$, Ho-Jin Shin, M.D. ${ }^{1}$, Goon-Jae Cho, M.D. ${ }^{1}$, Soo-Geun Wang, M.D. ${ }^{2}$, Byung-Joo Lee, M.D. ${ }^{2}$, Byung-Mann Cho, M.D. ${ }^{3}$, Dong-Won Kim, M.D. ${ }^{4}$, Hak-Jin Kim, M.D. ${ }^{5}$, Won Sik Lee, M.D. ${ }^{6}$, Young-Don Joo, M.D. ${ }^{6}$ and Chang-Hak Sohn, M.D. ${ }^{6}$

Departments of ${ }^{1}$ Internal Medicine, ${ }^{2}$ Otolaryngology, ${ }^{3}$ Preventive Medicine, ${ }^{4}$ Radiation Oncology, ${ }^{5}$ Radiology, Pusan National University College of Medicnce, ${ }^{6}$ Department of Internal Medicine, Inje University Hospital, Busan, Korea

Purpose: Although concurrent chemoradiotherapy (CCRT) has been considered as a standard treatment for locally advanced squamous cell carcinoma of the head and neck (SCCHN), this treament is associated with increased toxicities such as mucositis and dermatitis. As a result, the dose intensity can be reduced and interruptions of radiotherapy are more common for CCRT than for sequential treatment, especially for the elderly patients. This prospective study was performed to assess the efficacy and safety profiles of the induction chemotherapy of docetaxel and cisplatin for elderly patients with locally advanced SCCHN.

Materials and Methods: Patients over 65 years of age with locally advanced SCCHN were treated with docetaxel $\left(70 \mathrm{mg} / \mathrm{m}^{2}\right)$ and cisplatin $\left(75 \mathrm{mg} / \mathrm{m}^{2}\right)$ every 21 days. The chemotherapy consisted of two cycles with a third cycle that was administered to the responding patients. Patients who did not respond to initial chemotherapy underwent radiotherapy as a definitive local treatment.

\section{INTRODUCTION}

The majority of the 500,000 worldwide, annual, new case of squamous cell carcinoma of the head and neck (SCCHN) generally present with locally advanced disease $(1,2)$. As these patients' life expectancy increases, clinicians frequently confront the problems of treating elderly patients with cancer, including those with head and neck cancer. For example, almost

Correspondence: Jooseop Chung, Division of Hematology-oncology, Department of Internal Medicine, Pusan National University Hospital, 1-10, Ami-dong, Seo-gu, Busan 602-739, Korea. (Tel) 82-

51-240-7242, (Fax) 82-51-254-3127, (E-mail) hemon@pusan.ac.kr Received January 8, 2007, Accepted March 22, 2007

This work was supported for two years by Pusan National University Research Grant.
Results: Fifty patients were enrolled in this study and 44 patients were assessable for response and toxicity. The overall response rate was $88 \%, 16$ patients $(36 \%)$ achieved a complete response and 23 patients (52\%) achieved a partial response. After a median follow-up of 24 months (range: $9 \sim 38$ months) the median disease free period and overall survival period had not yet been reached. The one year and two year survival rates were $89 \%$ and $70 \%$, respectively. The most common grade $3 / 4$ adverse event was neutropenia, which occurred in 33 patients (75\%) and 4 patients had febrile neutropenia.

Conclusion: Combination chemotherapy of docetaxel and cisplatin is an effective regimen with an acceptable safety profile as the induction treatment for elderly patients suffering with SCCHN. (Cancer Res Treat. 2007; 39:1-5)

Key Words: Docetaxel, Cisplatin, Head and neck cancer, Induction chemtherapy

half of the approximately 40,000 new cases of SCCNH that are diagnosed annually in United States occur in the population older than 65 years (3).

On the Meta-Analysis of Chemotherapy for Head and Neck Cancer (MACH-NC 1 and MACH-NC2), concurrent chemoradiotherapy (CCRT) has shown a significant result; adding chemotherapy to radiation provides an $8 \%$ absolute survival benefit at 5 years (4). Consequently, CCRT has been recommended as a standard treatment for locally advanced SCCHN, but most clinical trials of CCRT have shown increased toxicities such as mucositis and dermatitis (5). As a result, CCRT causes a greater reduction of dose intensity and more common interruption of treatment than that for sequential treatment, especially for the elderly patients (6). So, it may be valuable to evaluate the efficacy of a new regimen of induction chemotherapy for elderly patients with SCCHN.

Several studies $(7 \sim 9)$ have demonstrated that cisplatin and fluorouracil (PF) induction chemotherapy results in significant cytoreduction for locally advanced SCCHN, as evidenced by 
the complete remission (CR) and overall response (OR) rates of $30 \%$ and $80 \%$, respectively. But PF combination therapy is complicated by mucosal toxicity and a prolonged infusion time.

The regimens including docetaxel have been reported on several clinical trials, and these regimens have a considerable effect for the patients with SCCNH $(10,11)$. This prospective study was performed to assess the efficacy and safety profiles of the induction chemotherapy of docetaxel and cisplatin for elderly patients with locally advanced SCCHN.

\section{MATERIALS AND METHODS}

\section{1) Patient eligibility}

The patients who were eligible for this study were required to meet the following criteria: they had histologically proven SCCHN except nasopharyngeal carcinoma, a completely unresectable tumor status, clinically and/or radiologically measurable disease as assessed by the Response Evaluation Criteria in Solid Tumors (RECIST) (12), no distant metastasis, no previous chemotherapy or radiotherapy, an Eastern Cooperative Oncology Group (ECOG) performance status of 0 or 1 , an age from 65 to 79 years old, adequate bone marrow reserves (an absolute neutrophil count $\geq 1.5 \times 10^{9} / \mathrm{L}$ and a platelet count $\geq$ $100 \times 10^{9} / \mathrm{L}$ ), adequate hepatic function (total bilirubin level $<$ $1.5 \mathrm{mg} / \mathrm{ul}$ and serum levels of asparate aminotransferase and alanine aminotransferase $<2.5 \times$ the upper normal limit) and adequate renal function (a serum creatinine level $<1.5 \mathrm{mg} / \mathrm{dl}$ ).

The exclusion criteria included the following: known hypersensitivity to drugs, serious concomitant illness (cirrhosis, ischemic heart disease or a bleeding tendency), neuropathy $>$ grade 2 according to the National Cancer Institute Common Toxicity Criteria (NCI-CTC) version 2.0, (13) active infection and active concomitant malignancy.

The stage of disease was defined by the American Joint Committee on Cancer (AJCC) criteria.

This study was reviewed and approved by our institutional review board and written informed consent was obtained from each patient.

\section{2) Treatment plan}

Docetaxel was administered at a dose of $70 \mathrm{mg} / \mathrm{m}^{2}$ over 1 hour, followed by hydration with a 2 hour infusion of 1,000 $\mathrm{ml}$ normal saline. Afterward, cisplatin was given at $75 \mathrm{mg} / \mathrm{m}^{2}$. Prophylactic corticosteroids were given with each dose of docetaxel to minimize the hypersensitivity reaction and peripheral edema.

The chemotherapy was repeated every 21 days if patient had recovered hematologically $\left(\mathrm{ANC} \geq 1.5 \times 10^{9} / \mathrm{L}\right.$, platelet $\geq 100$ $\times 10^{9} / \mathrm{L}$ ). The induction chemotherapy consisted of two cycles. If a response was recognized (complete response, CR or partial response, PR), then they received one more course of chemotherapy before undergoing the radiotherapy or an operation as radical local treatment.

Dose adjustments were permitted for both drugs according to the specific toxicities as noted in previous studies $(17 \sim 19)$. Colony stimulating factor was given for treating patients with grade 4 neutropenia or febrile neutropenia, for which there was a $25 \%$ subsequent dose reduction of both drugs. Complete blood counts were performed weekly and follow-up physical examinations, serum biochemistry, urine analysis and evaluation of the local findings were performed before each cycle of chemotherapy. CT or MRI scan and laryngoscopy were performed after the second cycle. Toxicity was graded according to the NCI-CTC version 2.0 criteria and the treatment response was assessed by the RECIST criteria.

\section{3) Statistical analysis}

The primary end point was the overall response rate after induction chemotherapy; the secondary objectives were toxicity evaluation and analysis of the response rate according to the disease site, stage and nodal status. The sample size was planned on the expected response rate of $85 \%$ with an allowable error of $10 \%$ and a $95 \%$ confidence interval (CI). The required number of patients was 49 . All the patients who received at least 2 cycles of treatment were assessable for their response. Chi-square tests were used for comparisons of the results. The significance level was defined as $p \leq 0.05$.

\section{RESULTS}

Fifty patients were enrolled in this study between April 2003 and March 2006. The median patient age was 69 years old (range: 65 79). The baseline characteristics of the study

Table 1. Patient characteristics

\begin{tabular}{lc}
\hline & No. $(\%)$ \\
\hline Patients & 50 \\
Gender, male : female & $47: 3$ \\
Age, years & \\
$\quad$ Median, range & $69,65 \sim 79$ \\
Primary tumor site & \\
$\quad$ Oropharynx & $16(32)$ \\
$\quad$ Hypopharynx & $11(22)$ \\
Larynx & $20(40)$ \\
$\quad$ Oral cavity & $3(8)$ \\
Performance status & \\
ECOG 0 & $26(52)$ \\
ECOG 1 & $24(48)$ \\
Stage & $31(62)$ \\
III & $19(38)$ \\
IV & \\
\hline
\end{tabular}

Table 2. TNM stage $(\mathrm{n}=50)$

\begin{tabular}{lrrrrr}
\hline & T1 & T2 & T3 & T4 & Overall \\
\hline N0 & & & 11 & 1 & 12 \\
N1 & 4 & 10 & 6 & 2 & 22 \\
N2 & 3 & 5 & 5 & 1 & 14 \\
N3 & 0 & 0 & 1 & 1 & 2 \\
Overall & 7 & 15 & 23 & 5 & 50 \\
\hline
\end{tabular}


Young-Jin Choi, et al : Docetaxel \& CDDP for Elderly SCCHN 3

Table 3. Response rate according to the primary disease site $(\mathrm{n}=44)$

\begin{tabular}{lrrrr}
\hline & & \multicolumn{3}{c}{ No. of patient $(\%)$} \\
\cline { 3 - 5 } $\begin{array}{l}\text { Disease } \\
\text { site }\end{array}$ & $\begin{array}{c}\text { No. of } \\
\text { patient }\end{array}$ & CR* & PR $^{+}$ & $\begin{array}{r}\text { Overall } \\
\text { response }\end{array}$ \\
\hline Oral cavity & 3 & $1(33)$ & $2(67)$ & $3(100)$ \\
Oropharynx & 14 & $5(33)$ & $8(50)$ & $13(93)$ \\
Larynx & 18 & $7(40)$ & $8(44)$ & $15(83)$ \\
Hypopharynx & 9 & $3(33)$ & $5(56)$ & $8(89)$ \\
Overall & 44 & $16(36)$ & $23(52)$ & $39(88)$ \\
\hline
\end{tabular}

${ }^{*}$ complete remission, ${ }^{\dagger}$ partial remission.

population are summarized in Table 1 and the stages of disease are presented as the tumor status and lymph node status in Table 2 . Thirty one patients $(62 \%)$ had stage III disease and 19 patients (38\%) had stage IV disease. Five patients had a T4 status and 2 patients had a N3 nodal status.

Of the 50 patients, 44 patients were assessable for the toxicities and the response of induction chemotherapy. Three patients underwent surgical treatment after the first cycle of chemotherapy without adequate evaluation and 2 patients refused any more chemotherapy just after the first cycle. One patient was dropped from the study due to late detection of concomitant esophageal cancer after the first cycle.

The OR rate of the evaluable patients was $88 \%$ (95\% CI: 75.4 96.2). A CR was achieved by 16 patients (36\%) and a PR was achieved by 23 (52\%) of 44 patients. Stable disease (SD) and progressive disease (PD) were shown for 3 patients and 2 patients, respectively. From the intent to treat analysis, the CR rate was 32\% (16 of 50) and the overall RR was $78 \%$ (39 of 50). The response rates according to the primary site are presented in Table 3 . There was no significant difference of the response rate according to the primary sites.

Of the 12 patients without nodal lesion, 7 patients (58\%) achieved a CR. Of the 32 patients with nodal metastasis, a CR was achieved by 9 (28\%) and a PR was achieved by 19 (59\%). The CR rate of the patients without nodal lesion was higher $(58 \%)$ than that of the patients with nodal lesion, but the difference did not reach statistical significance $(p=0.065)$. Seventeen patients $(85 \%)$ of 20 patients over 70 years old achieved either a CR or PR, and this was similar to the $65 \sim 70$ year old group (92\%) ( $\mathrm{p}=0.624)$.

Toxicity was analyzed in the 44 patients and this is presented in Table 4. A total of 127 cycles of chemotherapy were analyzed. Nine patients required a delay of their scheduled chemotherapy by a mean of one week due to myelosuppression $(n=5)$, fatigue $(n=3)$ and stomatitis $(n=1)$. A dose reduction of docetaxel by $25 \%$ was done for 8 patients and $25 \%$ attenuation of the cisplatin dose was done for 6 patients. The median dose intensity of docetaxel was $22.1 \mathrm{mg} / \mathrm{m}^{2} /$ week and the median dose of cisplatin was $23.8 \mathrm{mg} / \mathrm{m}^{2} /$ week. The mean relative dose intensity for docetaxel and cisplatin was 0.94 (range: $0.7 \sim 1.0$ ) and 0.95 (range: $0.7 \sim 1.0$ ), respectively. Granulocyte-colony stimulating factor was administered to 11 patients. The main adverse events were hematologic toxicity. The most common
Table 4. Toxicity of chemotherapy $(n=44)$

\begin{tabular}{lrrrr}
\hline \multicolumn{1}{c}{ Toxicity } & G1 & G2 & G3 & G4 \\
\hline Hematologic & & & & \\
$\quad$ Neutropenia & 2 & 9 & 22 & 11 \\
$\quad$ Anemia & 15 & 14 & 2 & 0 \\
Thrombocytopenia & 4 & 4 & 2 & 0 \\
Non-hematologic & & & & \\
$\quad$ Nausea/vomiting & 13 & 9 & 4 & 1 \\
Fatigue & 16 & 7 & 8 & 0 \\
Anorexia & 18 & 5 & 5 & 0 \\
Neurotoxicity & 5 & 4 & 1 & 0 \\
Stomatitis & 11 & 9 & 2 & 0 \\
Diarrhea & 4 & 3 & 2 & 0 \\
Renal toxicity & 2 & 2 & 0 & 0 \\
\hline
\end{tabular}

toxicity of $\geq$ Grade 3 was neutropenia, which occurred in 33 patients (75\%) and 4 of these patients had febrile neutropenia. The neutropenia was reversible. The median time to the nadir of neutropenia was 9 days (range: $5 \sim 15$ days). The median recovery time to grade 0 was 8 days (range: $4 \sim 15$ days). There was no treatment-related death. Anemia occurred in 31 patients (70\%), but anemia $\geq$ Grade 3 was noted in only 2 patients (5\%). The non-hematologic toxicities were generally tolerable. The common toxicities of $\geq$ Grade 3 were fatigue in 8 patients $(18 \%)$, emesis in 5 patients $(11 \%)$, anorexia in 5 patients $(11 \%)$ and stomatitis in 2 patients (5\%). Renal toxicities were noted in 4 patients and this resulted in dose reduction of cisplatin for 2 patients with grade 2 renal toxicities.

\section{DISCUSSION}

Induction chemotherapy for locally advanced SCCHN has been studied for the past 3 decades $(7 \sim 9)$. There are a number of problems with administering induction chemotherapy for SCCHN patients such as cross resistance to RT and the controversy about the extent of surgical resection in the responders $(14,15)$. The increased toxicity and overall duration of treatment also seem to be disadvantages. However, previous studies have shown that induction chemotherapy yielded several advantages such as better drug delivery through an intact vasculature and the early treatment of micrometastasis (16). It has been confirmed that patients with $\mathrm{N} 2 \mathrm{C}$ and $\mathrm{N} 3$ nodal disease have greater rates of distant recurrence and this suggested that lowering the rate of metastasis by induction chemotherapy would likely improve survival (17), The response to induction chemotherapy can be a guide for further treatment after definitive local treatment, if it is needed. The MACH-NC showed an absolute survival benefit of $2 \%$ by administering neoadjuvant chemotherapy with PF and an $8 \%$ absolute survival benefit at 5 years with administering CCRT $(6,7)$.

As single agent, docetaxel yields a response rate ranging from $20 \%$ to $40 \%$ for metastatic SCCHN $(18,19)$. The activity and non-overlapping toxicities with platinum could allow this to be a good combination for SCCHN. It has been reported that a docetaxel and cisplatin regimen is an effective treatment, with 
an acceptable safety profile, for palliation of recurrent SCCHN and the results showed higher response and survival rates with a short duration of administration and a lower incidence of severe mucosal toxicity, as compared with PF $(10,11)$.

A Japanese phase I trial determined the maximal tolerable dose of docetaxel as $60 \sim 70 \mathrm{mg} / \mathrm{m}^{2}$ for the patients with SCCHN (20). So the present study was designed with using a dose of $75 \mathrm{mg} / \mathrm{m}^{2}$ of cisplatin and a $70 \mathrm{mg} / \mathrm{m}^{2}$ dose of docetaxel, which was lower than the $75 \sim 100 \mathrm{mg} / \mathrm{m}^{2}$ dose of docetaxel used in western countries.

Studies on adding docetaxel to PF have shown high activity for SCCHN (OR: 71 100\%). A phase III study showed significant better overall survival in the docetaxel induction arm than in the PF arm (18.6 vs 14.5 months, respectively) (21). But this docetaxel regimen required intensive supportive care and treatment delays. Furthermore, the toxicities must be worse for elderly patients with SCCHN, so this encourages physicians to administer more tolerable and safer treatment for elderly patients prior to definitive local treatment. Therefore, this clinical study was undertaken to assess the efficacy and toxicity of the docetaxel and cisplatin regimen for the elderly patients with SCCHN. The present study showed an $88 \%$ overall response rate, including a $36 \% \mathrm{CR}$ and tolerable toxicity. This result is not worse than those reported for the standard PF regimen for the general population, and it seems to be a good result for elderly patients. A CR to induction chemotherapy is predictive of improved survival (22). However, the present study was a phase II trial and it needs to be followed up to determine if the high response rate can result in survival improvement.

Before the era of taxane, induction treatment did not confer a survival benefit despite its high response $(8,9)$. However, the present result with including docetaxel in the regime offers an advantage over standard PF for the toxicity and convenience.

Although the incidence of severe neutropenia was high (75\%), only 4 patients (4\%) experienced neutropenic fever. It suggests that the patients' neutropenia recovered quite well. Anemia was a frequent adverse effect, but its grade was mild (grade 1 or grade 2). So it is probably wise to administer EPO if this treatment is planned for the elderly patients with SCCHN (23). The favorable toxicity of the current study can be explained by the good performance (ECOG 0 or 1 ) of the enrolled patients. But the combination of docetaxel and cisplatin can be administered during one day and this showed tolerable toxicity, and especially for the severe mucositis that is a major complication of the PF regimen. A treatment that dose not cause profound mucositis would be helpful to completely treat elderly patients with SCCHN, as compared with PF regimen., The convenience of a one day infusion every 21 days is also beneficial compared with the PF regimen.

\section{CONCLUSIONS}

The current study suggests that combination chemotherapy of docetaxel and cisplatin is an effective regimen with acceptable safety profiles as an induction treatment for the elderly patients with SCCHN. Yet it remains to be seen whether the high response rate results in improved survival.

\section{REFERENCES}

1. Parkin DM, Pisani P, Ferlay J. Estimates of the worldwide incidence of 25 major cancers in 1990. Int J Cancer. 1999; 80:827-41.

2. Zbaren P, Lehmann W. Frequency and sites of distant metastases in head and neck squamous cell carcinoma. Arch Otolaryngol Head Neck Surg. 1987;113:762-4.

3. Ries LA, Eisner MP, Kosary CL. SEER Cancer Statistics Review, 1975 2000. Bethesda, MD: National Cancer Institute; 2003.

4. Pignon JP, Bourhis J, Domenge C, Designe L. Chemotherapy added to locoregional treatment for head and neck squamouscell carcinoma: Three meta-analyses of updated individual data. MACH-NC collaborative group. Meta-analysis of chemotherapy on head and neck cancer. Lancet. 2000;355:949-55.

5, CohenEE, Lingen MW, Vokes EE. The expanding role of systemic therapy in head and neck cancer. J Clin Oncol. 2004; 22:1743-52.

6. Vokes EE, Stenson K, Rosen FR, Kies MS, Rademaker AW, Witt ME, et al. Weekly carboplatin and paclitaxel followed by concomitant paclitaxel, fluorouracil, and hydroxyurea chemoradiotherapy: curative and organ-preserving therapy for advanced head and neck cancer. J Clin Oncol. 2003;21:320-6.

7. Lefebvre JL, Chevalier D, Luboinski B, Kirkpatrick A, Collette L, Sahmoud T. Larynx preservation in pyriform sinus cancer: preliminary results of a European organization for research and treatment of cancer phase III trial-EORTC head and neck cancer cooperative group. J Natl Cancer Inst. 1996;88:890-9.

8. Licitra L, Grandi C, Guzzo M, Mariani L, Lo Vullo S, Valvo $F$, et al. Primary chemotherapy in resectable oral cavity squamous cell cancer: a randomized controlled trial. J Clin Oncol. 2003;21:327-33.

9. Domenge C, Hill C, Lefebvre JL, De Raucourt D, Rhein B, Wibault $\mathrm{P}$, et al. Randomized trial of neoadjuvant chemotherapy in oropharyngeal carcinoma: French Groupe d'Etude des Tumeurs de la Tete et du Cou (GETTEC). Br J Cancer. 2000;83:1594-8.

10. Schöffski P, Catimel G, Planting AS, Droz JP, Verweij J, Schrijvers D, et al. Docetaxel and cisplatin: an active regimen in patients with locally advanced, recurrent or metastatic squamous cell carcinoma of the head and neck. Results of a phase II study of the EORTC early clinical studies group. Ann Oncol. 1999;10:119-22.

11. Glisson BS, Murphy BA, Frenette G, Khuri FR, Forastiere AA. Phase II trial of docetaxel and cisplatin combination chemotherapy in patients with squamous cell carcinoma of the head and neck. J Clin Oncol. 2002;20:1593-99.

12. Therasse P, Arbuck SG, Eisenhauer EA, Wanders J, Kaplan RS, Rubinstein L, et al. New guidelines to evaluate the response to treatment in solid tumors. J Natl Cancer Inst. 2000; 92:205-16.

13. National Cancer Institute. National Cancer Institute Common Toxicity Criteria. [On-line]. http://ctep.info.nih.gov/reporting/ index.html

14. Ozols RF, Masuda H, Hamilton TC. Mechanisms of crossresistance between radiation and antineoplastic drugs. NCI Monogr. 1988;6:159-65.

15. Ensley JF, Jacobs JR, Weaver A, Kinzie J, Crissman J, Kish JA, et al. Correlation between response to cisplatinum-combination chemotherapy and subsequent radiotherapy in previously untreated patients with advanced squamous cell can- 
cers of the head and neck. Cancer. 1984;54:811-4.

16. Browman GP. Evidence-based recommendations against neoadjuvant chemotherapy for routine management of patients with squamous cell head and neck cancer. Cancer Invest. 1994;12:662-70.

17. Brockstein B, Haraf DJ, Rademaker AW, Kies MS, Stenson $\mathrm{KM}$, Rosen F, et al. Patterns of failure, prognostic factors and survival in locoregionally advanced head and neck cancer treated with concomitant chemoradiotherapy: a 9-year, 337patient, multi-institutional experience. Ann Oncol. 2004;15: 1179-86.

18. Dreyfuss AI, Clark JR, Norris CM, Rossi RM, Lucarini JW, Busse PM, et al. Docetaxel: an active drug for squamous cell carcinoma of the head and neck. J Clin Oncol. 1996;14: $1672-8$.

19. Catimel G, Verweij J, Mattijssen V, Hanauske A, Piccart M, Wanders J, et al. Docetaxel (Taxotere): an active drug for the treatment of patients with advanced squamous cell carcinoma of the head and neck. EORTC early clinical trials group. Ann Oncol. 1994;5:533-7.

20. Colevas AD, Busse PM, Norris CM, Fried M, Tishler RB, Poulin M, et al. Induction chemotherapy with docetaxel, cis- platin, fluorouracil, and leucovorin for squamous cell carcinoma of the head and neck: a phase I/II trial. J Clin Oncol. 1998;16:1331-9.

21. Vermorken JB, Remenar E, Van Herpen C. Standard cisplatin/ infusional 5-fluorouracil (PF) vs docetaxel (T) plus PF (TPF) as neoadjuvant chemotherapy for nonresectable locally advanced squamous cell carcinoma of the head and neck (LA-SCCHN): a phase III trial of the EORTC head and neck cancer group. Proc Am Soc Clin Oncol. 2004;23 (abstr 5508).

22. Clavel M, Vermorken JB, Cognetti F, Cappelaere P, de Mulder $\mathrm{PH}$, Schornagel JH, et al. Randomized comparison of cisplatin, methotrexate, bleomycin and vincristine (CABO) versus cisplatin and 5-fluorouracil (CF) versus cisplatin (C) in recurrent or metastatic squamous cell carcinoma of the head and neck. A phase III study of the EORTC head and neck cancer cooperative group. Ann Oncol. 1994;5:521-6.

23. Gebbia V, Di Marco P, Citarrella P. Systemic chemotherapy in elderly patients with locally advanced and/or inoperable squamous cell carcinoma of the head and neck: impact of anemia and role of recombinant human erythropoietin. Crit Rev Oncol Hematol. 2003;48(Suppl):S49-55. 